\title{
Reaction Chemistry of ZnTe Metalorganic Vapor-Phase Epitaxy
}

\author{
Kerri J. Wilkerson, Menno J. Kappers, and Robert F. Hicks* \\ Department of Chemical Engineering, 5531 Boelter Hall, University of California at Los Angeles, \\ Los Angeles, California 90095-1592 \\ Received: December 4, 1996; In Final Form: January 30, $1997^{\otimes}$
}

\begin{abstract}
The reaction chemistry of zinc telluride (ZnTe) metalorganic vapor-phase epitaxy (MOVPE) from dimethylzinc (DMZn), diethylzinc (DEZn), and diisopropyltelluride (DIPTe) has been studied using on-line gas chromatography and infrared spectroscopy. Two growth regimes have been discovered: one at low values of the II/VI ratio and the other at higher values of the II/VI ratio. In the first regime, the group VI compound is consumed in excess, while in the second regime, the group II compound is consumed in excess. The crossover point lies at II/VI $=5.0$ for DMZn and at II/VI $=0.3$ for DEZn. Stoichiometric ZnTe is deposited under all growth conditions. The excess DIPTe consumed is converted into volatile diisopropylditelluride and isopropyltellurol. Conversely, the excess DEZn or DMZn consumed produces zinc metal. These waste byproducts accumulate in the outlet of the reactor. The hydrocarbon products generated from the ethyl and isopropyl ligands are indicative of radical disproportionation, recombination, and hydrogenation reactions. However, the methyl ligands mainly undergo surface hydrogenation to produce methane.
\end{abstract}

\section{Introduction}

The wide bandgap of zinc telluride $\left(E_{\mathrm{g}}=2.26 \mathrm{eV}\right)$ makes it a useful material in several applications, such as the first unit in a tandem solar cell, as a buffer layer for an $\mathrm{HgCdTe}$ infrared detector, or as part of the graded $\mathrm{p}-\mathrm{Zn}(\mathrm{Te}) \mathrm{Se}$ multiquantumwell structure in a blue-green laser diode. ${ }^{1-7}$ Metalorganic vapor-phase epitaxy (MOVPE) is an attractive technology for large-scale production of these devices. ${ }^{1,5,7-11}$ However, the MOVPE of ZnTe is not completely understood at this time. Information is lacking on the reactions that occur during film growth and on the products other than $\mathrm{ZnTe}$ that are exhausted from the reactor.

Prior research on ZnTe MOVPE has focused on monitoring the film growth rate as a function of the process conditions. ${ }^{1-4,7,8}$ Apparent activation energies for ZnTe MOVPE were obtained ranging from 60 to $125 \mathrm{~kJ} \mathrm{~mol}^{-1}$ for a number of different precursors and at temperatures between 623 and $723 \mathrm{~K} \cdot 3,7,10$ These results indicate that the process is kinetically limited and occurs via a heterogeneous reaction pathway. Additional kinetic information can be obtained from monitoring not only the film growth rate but also the consumption rates of the organometallic sources and the production rates of the hydrocarbon products. This approach has been used by us to study CdTe MOVPE. ${ }^{10}$ We found that the organometallic precursors adsorb onto the semiconductor surface and decompose via the desorption, recombination, hydrogenation, and disproportionation of the alkyl ligands. Alkyls that desorb as radicals undergo recombination, hydrogenation, and disproportionation in the gas as well. We also discovered that, at a II/VI ratio higher than 0.5 , the growth rate is limited by the group VI compound, whereas at a II/VI ratio lower than 0.5 , the group II source is rate-limiting. In the regime where the group VI source limits the deposition rate, the $\mathrm{Cd}$ atoms produced in excess of that needed for CdTe film growth are desorbed from the surface and deposit as a metal film or fly ash in the reactor outlet. We were curious whether this also occurs during ZnTe MOVPE and have therefore studied this reaction in detail.

In this paper we report on the reaction chemistry of $\mathrm{ZnTe}$

\footnotetext{
* To whom correspondence should be addressed.

${ }^{\otimes}$ Abstract published in Advance ACS Abstracts, March 1, 1997.
}

MOVPE using dimethylzinc (DMZn), diethylzinc (DEZn), and diisopropyltelluride (DIPTe). The deposition process has been monitored by infrared spectroscopy (IR) and gas chromatography (GC) of the vapor feed and effluent from the reactor. Infrared spectroscopy is essential for characterizing both the reactants and products of ZnTe MOVPE. Moreover, our GC analysis of the hydrocarbons indicates that the DMZn and DEZn decompose by two distinctly different reaction pathways.

\section{Experimental Methods}

The experiments were carried out using an atmosphericpressure MOVPE reactor, which is described in detail elsewhere. ${ }^{10}$ The ZnTe was deposited on the inner walls of a glass tube, $0.4 \mathrm{~cm}$ in diameter, that narrowed to $0.2 \mathrm{~cm}$ in the reaction zone. The reactor temperature was maintained to within $2 \mathrm{~K}$ over the $5 \mathrm{~cm}$ length of the heated zone. Outside the heated region, the glass tube was rapidly quenched to $300 \mathrm{~K}$ with watercooled, heat exchangers. Electronic-grade DMZn, DEZn, and DIPTe were contained in $200 \mathrm{~cm}^{3}$ stainless steel bubblers immersed in thermal regulating baths at 258, 288, and $306 \mathrm{~K}$, respectively. The precursors were vaporized into $0.5-30 \mathrm{~cm}^{3}$ $\mathrm{min}^{-1}$ of helium, then diluted with either hydrogen or helium to between 300 and $600 \mathrm{~cm}^{3} \mathrm{~min}^{-1}$, and fed to the reactor. The $\mathrm{H}_{2}$ and $\mathrm{He}(99.995 \%)$ were purified by deoxygenation traps and $13 \mathrm{X}$ molecular sieves held at $195 \mathrm{~K}$.

Gas from the MOVPE system was sampled with a HewlettPackard 5890A gas chromatograph equipped with a flameionization detector and a $30 \mathrm{~m}$ long, megabore capillary column containing a $1 \mu \mathrm{m}$ thick, stationary phase of poly(methylsiloxane) (Restek $\left.\mathrm{Rt}_{\mathrm{x}}-1\right)$. During growth, the inlet and outlet gas compositions were measured, and the difference in the integrated peak areas was taken as a measure of the production rate of the hydrocarbons. The GC sampling technique was configured for the analysis of hydrocarbons only, since the zinc precursors decomposed in the capillary column. The unreacted organometallic molecules were frozen in a region of the capillary tubing by holding it at $185 \mathrm{~K}$. At this temperature, the hydrocarbons passed on into the column. Next, the column was held at 218 $\mathrm{K}$ for $2.3 \mathrm{~min}$, ramped to $353 \mathrm{~K}$ at a rate of $30 \mathrm{~K} \mathrm{~min}^{-1}$, and held at $353 \mathrm{~K}$ for $0.2 \mathrm{~min}$. Once the hydrocarbons eluted from 
TABLE 1: Assignment of the Vibrational Bands Observed for DIPTe, DMZn, and DEZn in the Region between 1600 and 450 $\mathrm{cm}^{-1}(\text { Refs 17-19) })^{a}$

\begin{tabular}{|c|c|c|c|c|c|}
\hline \multicolumn{2}{|c|}{ DIPTe } & \multicolumn{2}{|c|}{$\mathrm{DMZn}$} & \multicolumn{2}{|c|}{ DEZn } \\
\hline freq & assignt & freq & assignt & freq & assignt \\
\hline 504 (vw) & $v(\mathrm{M}-\mathrm{C})$ & $604(\mathrm{~s})$ & $v(\mathrm{M}-\mathrm{C})$ & $572(\mathrm{~s})$ & \multirow{4}{*}{$\begin{array}{l}v(\mathrm{M}-\mathrm{C}) \\
\rho\left(\mathrm{CH}_{2}\right)\end{array}$} \\
\hline 874 (vw) & \multirow{2}{*}{\}$v(\mathrm{C}-\mathrm{C}-\mathrm{C})$} & $621(\mathrm{~s})$ & \multirow[t]{2}{*}{$v(\mathrm{M}-\mathrm{C})$} & 612 (br) & \\
\hline $923(\mathrm{vw})$ & & & & & \\
\hline $1000(w)$ & $\rho\left(\mathrm{CH}_{3}\right)$ & 706 (br) & $\rho\left(\mathrm{CH}_{3}\right)$ & & \\
\hline 1019 (vw) & \multirow{6}{*}{$v(\mathrm{C}-\mathrm{C}) / \rho\left(\mathrm{CH}_{3}\right)$} & & & 957 (vw) & \multirow{3}{*}{$v(\mathrm{C}-\mathrm{C}) / \rho\left(\mathrm{CH}_{3}\right)$} \\
\hline $1148(\mathrm{~s})$ & & & & $986(\mathrm{~m})$ & \\
\hline \multirow[t]{4}{*}{1197 (s) } & & & & 1944 (vw) & \\
\hline & & & & $1188(\mathrm{w})$ & $\delta\left(\mathrm{CH}_{3}\right)$ \\
\hline & & & & 1234 (vw) & $\omega\left(\mathrm{CH}_{2}\right)$ \\
\hline & & $1030(\mathrm{vw})$ & $\mathrm{c} / \mathrm{o}$ & $1376(\mathrm{sh})$ & $\mathrm{c} / \mathrm{o}$ \\
\hline $1303(w)$ & \multirow{3}{*}{$\delta \delta\left(\mathrm{CH}_{3}\right)$} & 1175 (vw) & 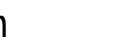 & $1383(\mathrm{~m})$ & \multirow{3}{*}{$\delta\left(\mathrm{CH}_{x}\right)$} \\
\hline $1372(\mathrm{~m})$ & & $1192(w)$ & $\delta\left(\mathrm{CH}_{3}\right)$ & $1421(w)$ & \\
\hline $1462(\mathrm{~m})$ & & $1304(w)$ & & $1469(w)$ & \\
\hline
\end{tabular}

${ }^{a}$ Abbreviations: $\mathrm{vw}=$ very weak, $\mathrm{w}=$ weak, $\mathrm{m}=$ medium, $\mathrm{s}=$ strong, $\mathrm{br}=$ broad, $\mathrm{sh}=$ shoulder, $\mathrm{c} / \mathrm{o}=$ combination/overtone.

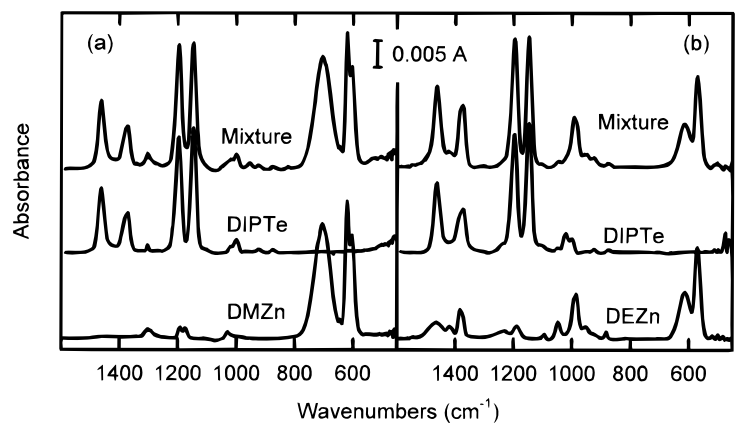

Figure 1. Infrared spectra of the organometallic precursors alone and mixed: (a) $500 \mathrm{ppm}$ DMZn and 500 ppm DIPTe; (b) 500 ppm DEZn and $500 \mathrm{ppm}$ DIPTe.

the column, the column was taken out of line to the flameionization detector (FID). The cold region was then ramped to room temperature, and the organometallic compounds were injected directly into the FID.

The consumption rates of DMZn, DEZn, and DIPTe were determined by monitoring the gas-phase composition of the reactor feed and effluent with an infrared spectrometer. The data were acquired with a BioRad FTS-7 spectrometer and an Infrared Associates wide-band MCT detector. Infrared spectra were collected of the gas streams by passing them through a small flow cell, $17.7 \mathrm{~cm}$ long and $1.6 \mathrm{~cm}$ in diameter, and sealed with $\mathrm{KBr}$ windows. Adequate signal to noise was obtained by coadding 64 scans at $4 \mathrm{~cm}^{-1}$ resolution.

Shown in Figure 1 are infrared spectra of the individual organometallic compounds and of a mixture of each. The spectrum of the mixture is an exact summation of the spectra of the individual organometallic compounds. Listed in Table 1 are the assignments of the absorption bands for DIPTe, DMZn, and DEZn observed between 1600 and $450 \mathrm{~cm}^{-1} \cdot{ }^{17-19}$ Relatively intense bands are detected at $621 \mathrm{~cm}^{-1}$ for DMZn and $572 \mathrm{~cm}^{-1}$ for DEZn, which correspond to the asymmetric metal-carbon stretching vibrations. For DIPTe, there is an intense skeletal mode associated with the isopropyl ligand at $1148 \mathrm{~cm}^{-1}$. These bands are well-resolved from each other and were chosen as a means of independently monitoring the consumption of each molecule during MOVPE.

The intensities of the selected infrared bands were calibrated against the concentrations of the organometallic compounds as follows. The precursor concentrations were determined with gas chromatography by measuring the hydrocarbons produced during complete pyrolysis of each organometallic compound at $900 \mathrm{~K}$. The hydrocarbons were summed and converted to a molar concentration of the precursor using the ideal gas law.

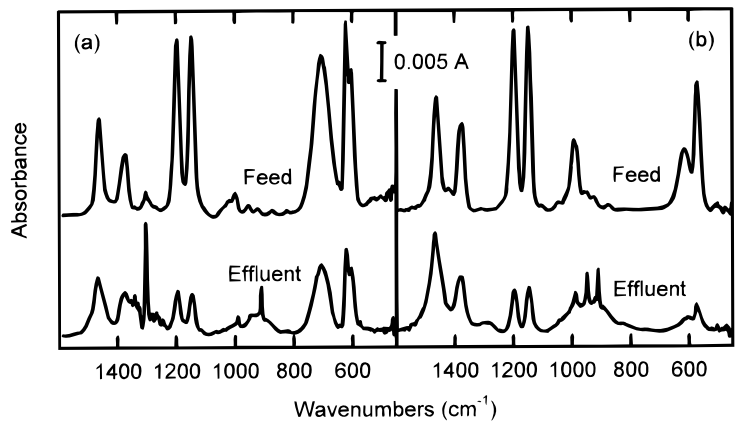

Figure 2. Infrared spectra of the reactor feed and effluent during MOVPE in $\mathrm{H}_{2}$ at $713 \mathrm{~K}$ and a total flow rate of $200 \mathrm{~cm}^{3} \mathrm{~min}^{-1}$ from (a) DMZn and DIPTe and (b) DEZn and DIPTe.

Then a graph of the infrared absorbance versus the precursor concentration was prepared. From the slope of the resulting straight line, the molar absorption coefficient $(\epsilon)$ of the selected infrared bands was calculated using Beer's law. The calculated absorption coefficients are $(76 \pm 2) \times 10^{3},(57 \pm 2) \times 10^{3}$ and $(100 \pm 6) \times 10^{3} \mathrm{~cm}^{2} \mathrm{~mol}^{-1}$ for the DMZn infrared band at 621 $\mathrm{cm}^{-1}$, the DEZn infrared band at $572 \mathrm{~cm}^{-1}$, and the DIPTe infrared band at $1148 \mathrm{~cm}^{-1}$, respectively. Based on this analysis, the detection limit of the infrared technique is approximately $10 \mathrm{ppm}$ for each organometallic compound.

The consumption rates of the precursors were determined during ZnTe deposition by monitoring the change in the intensities of the infrared bands from the inlet to the outlet of the reactor. Shown in Figure 2 are samples of the feed and effluent gases during ZnTe MOVPE from DMZn and DIPTe (graph a) and DEZn and DIPTe (graph b) at $673 \mathrm{~K}$ and 200 $\mathrm{cm}^{3} \mathrm{~min}^{-1}$ total flow. Notice the decrease in the peak intensities of the organometallic compounds at the reactor outlet. In addition, during growth with DMZn, sharp bands appear at 1300 and $910 \mathrm{~cm}^{-1}$. These correspond to the $\mathrm{C}-\mathrm{H}$ bending mode of methane and to the in-phase $\mathrm{C}=\mathrm{CH}_{2}$ wagging mode of propylene, respectively. ${ }^{20-22}$ In the case of MOVPE with DEZn and DIPTe, the effluent gas produces absorption peaks at 910 and $950 \mathrm{~cm}^{-1}$ due to the wagging modes of propylene and ethylene.

The dependence of the $\mathrm{ZnTe}$ deposition rate, $r_{\mathrm{ZnTe}}$, on the surface temperature and the concentration of the organometallic reagents can be expressed as follows:

$$
r_{\mathrm{ZnTe}}=\frac{n}{N} A \exp \left(-\frac{E_{\mathrm{a}}}{R T}\right) P_{\mathrm{II}}^{u} P_{\mathrm{VI}}^{v}
$$

where $n$ is the number of adsorption sites $\left(\mathrm{cm}^{-2}\right), N$ is 


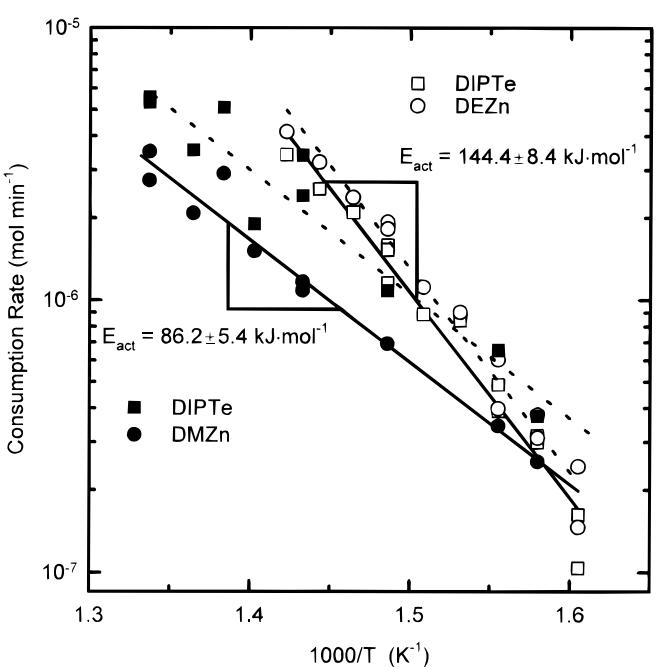

Figure 3. Dependence of the consumption rates of the organometallic compounds on inverse temperature for $\mathrm{ZnTe}$ MOVPE in $\mathrm{H}_{2}$ with DMZn, DEZn, and DIPTe. The II/VI ratio is 1.2 , the DMZn partial pressure is $4.8 \times 10^{-4} \mathrm{~atm}$, and the DEZn partial pressure is $4.0 \times$ $10^{-4} \mathrm{~atm}$.

Avogadro's number, $A$ and $E_{\mathrm{a}}$ are the apparent frequency factor $\left(\mathrm{s}^{-1}\right)$ and activation energy $\left(\mathrm{kJ} \mathrm{mol}^{-1}\right)$ for the reaction, $R$ is the ideal gas constant, $T$ is the temperature, $P_{\mathrm{II}}$ and $P_{\mathrm{VI}}$ are the fractional partial pressures of the group II and VI reactants, and $u$ and $v$ are the reaction orders for each reactant. The number of adsorption sites is assumed to be $5.4 \times 10^{14} \mathrm{~cm}^{-2}$, which is equivalent to 2 sites per $(6.10 \AA)^{2}$, where $6.10 \AA$ is the lattice constant for ZnTe. It is apparent from eq 1 that, in order to determine an accurate activation energy, the partial pressure of each reactant must remain constant within the reactor. Accordingly, the reactor was operated under conditions where the conversion of the limiting reagent was held to less than $10 \%$. This was accomplished by offsetting any increase in reaction rate with an increase in the total gas flow rate.

Periodically, the mass of the film deposited was determined by measuring the weight of the tube before and after growth. This value matched to within $10 \%$ of the mass calculated from the consumption rate of the limiting organometallic reagent and the time of deposition. During growth, the production rate of the hydrocarbons measured by gas chromatography was compared to the consumption rate of the organometallic precursors measured by infrared spectroscopy. The production rate of $\mathrm{C}_{3}$ to $\mathrm{C}_{6}$ hydrocarbons yielded an equivalent consumption rate of DIPTe that was up to $50 \%$ less than that estimated from the infrared data. This discrepancy suggested that not all the propylcontaining reaction products were detected by gas chromatography. Sometimes the zinc sources decomposed in the capillary lines leading to the GC and increased the amount of methane detected from DMZn or the amount of ethane detected from DEZn, above that generated in the MOVPE reactor. Whenever this occurred, the amount of methane and ethane recorded was decreased so as to close the zinc mass balance. This seemed valid since the consumption rates determined by IR spectroscopy accurately predicted the film mass.

\section{Results}

Consumption of the Organometallic Compounds. Shown in Figure 3 is an Arrhenius plot for ZnTe MOVPE in $\mathrm{H}_{2}$ from DMZn and DIPTe (filled symbols). The partial pressure of DMZn in the inlet is $4.8 \times 10^{-4} \mathrm{~atm}$, and the II/VI ratio is 1.2. The apparent activation energy for ZnTe deposition is $86.2 \pm$ $5.4 \mathrm{~kJ} \mathrm{~mol}^{-1}$, as determined from the slope of the line for DMZn

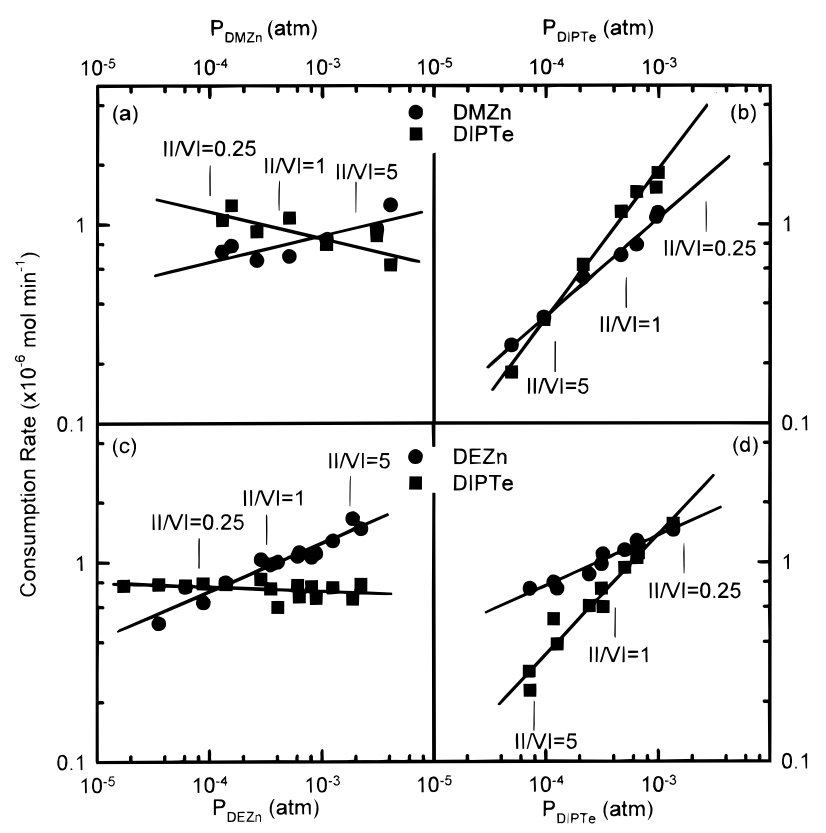

Figure 4. Dependence of the consumption rates of sources on their partial pressures for $\mathrm{ZnTe}$ MOVPE in $\mathrm{H}_{2}$ at $673 \mathrm{~K}$ : (a) varying the DMZn pressure at $3.4 \times 10^{-4} \mathrm{~atm}$ of DIPTe, (b) varying the DIPTe pressure at $4.4 \times 10^{-4} \mathrm{~atm}$ of DMZn, (c) varying the DEZn pressure at $3.2 \times 10^{-4} \mathrm{~atm}$ of DIPTe, and (d) varying the DIPTe pressure at 4.0 $\times 10^{-4} \mathrm{~atm}$ of DEZn.

consumption (the limiting reagent). This value for $E_{\text {act }}$ is consistent with previously reported values for ZnTe MOVPE. ${ }^{3,7}$ The frequency factor is $1.9 \times 10^{7} \mathrm{~s}^{-1}$, as determined from the intercept of the line. The figure also reveals that at a II/VI ratio of 1.2 the DIPTe is consumed in excess of the DMZn throughout the temperature range studied. As the temperature of the reactor rises, the amount of excess DIPTe increases, ranging from 0.2 $\times 10^{-6} \mathrm{~mol} \mathrm{~min}^{-1}$ at $625 \mathrm{~K}$ to $3.0 \times 10^{-6} \mathrm{~mol} \mathrm{~min}^{-1}$ at $760 \mathrm{~K}$. This amount can be greater than $50 \%$ of the DMZn consumed.

Also shown in Figure 3 is an Arrhenius plot for $\mathrm{ZnTe}$ MOVPE using DEZn and DIPTe (open symbols). The concentration of DEZn is $4.1 \times 10^{-4}$ atm, and the II/VI ratio is 1.2. The growth rate of ZnTe with DEZn is higher than that with DMZn throughout most of the temperature range studied. The apparent activation energy and frequency factor for $\mathrm{ZnTe}$ deposition are $144.4 \pm 8.4 \mathrm{~kJ} \mathrm{~mol}^{-1}$ and $1.4 \times 10^{12} \mathrm{~s}^{-1}$, respectively. These values are determined from the slope and intercept of line for the DIPTe consumption (the limiting reagent). This apparent activation energy is slightly higher than that previously reported. ${ }^{3,7}$ In contrast to growth with DMZn, the DEZn is consumed in excess of the DIPTe at a II/VI ratio of 1.2. The amount of excess DEZn consumed increases with temperature, ranging from $0.5 \times 10^{-7} \mathrm{~mol} \mathrm{~min}^{-1}$ at $625 \mathrm{~K}$ to $9.2 \times 10^{-7} \mathrm{~mol} \mathrm{~min}^{-1}$ at $700 \mathrm{~K}$.

Shown in the upper half of Figure 4 are the dependencies of the consumption rates of DMZn and DIPTe on the partial pressures of each of these sources at $673 \mathrm{~K}$. On the left side of the figure (graph a), the partial pressure of DMZn is varied, while keeping DIPTe constant at $3.4 \times 10^{-4}$ atm. The consumption rate of DMZn exhibits a positive dependence on its partial pressure. The reaction order calculated from the slope of the line is 0.13 . In contrast, the consumption rate of DIPTe decreases with increasing DMZn pressure. The reaction order in this case is -0.13 . On the upper right side of Figure 4 (graph b), the partial pressure of DIPTe is varied, while keeping the DMZn constant at $4.4 \times 10^{-4} \mathrm{~atm}$. As the partial pressure of DIPTe increases, the consumption rates of the group II and VI sources rise by about 1 order of magnitude. The reaction orders 
TABLE 2: Kinetic Data for MOVPE of ZnTe in the Two Different Growth Regimes (Cf. Eq 1)

\begin{tabular}{lllrrrr}
\hline & & & \multicolumn{2}{c}{ order in partial pressure } & \\
\cline { 4 - 5 } II/VI & $E_{\text {act }}{ }^{a}$ & & \multicolumn{2}{c}{$u$} & $v$ & \\
\cline { 4 - 5 } ratio & $\left(\mathrm{kJ} \mathrm{mol}^{-1}\right)$ & $A^{a}\left(\mathrm{~s}^{-1}\right)$ & DMZn & DEZn & DIPTe & remark \\
\hline$<5.0$ & 86.2 & $1.9 \times 10^{7}$ & 0.13 & & 0.50 & excess DIPTe \\
$>5.0$ & $\mathrm{~nm}$ & $\mathrm{~nm}$ & -0.13 & & 0.75 & excess DMZn \\
$<0.3$ & $\mathrm{~nm}$ & $\mathrm{~nm}$ & & 0.24 & 0.26 & excess DIPTe \\
$>0.3$ & 144.4 & $1.4 \times 10^{12}$ & & -0.02 & 0.60 & excess DEZn
\end{tabular}

${ }^{a}$ Values measured at a II/VI ratio of 1.2. ${ }^{b}$ Values measured at 673 $\mathrm{K} ; \mathrm{nm}=$ not measured.

calculated from the slopes of the lines are 0.5 and 0.75 , respectively.

Further examination of Figure 4 reveals that the consumption rates of DMZn and DIPTe cross each other at a II/VI ratio near 5.0 (refer to Figure 4b). Below 5.0, DMZn is the limiting reagent so that its decomposition rate equals the rate of $\mathrm{ZnTe}$ film growth. Conversely, above a II/VI ratio of 5.0, the limiting reagent is DIPTe, and its conversion determines the rate of $\mathrm{ZnTe}$ deposition. In other words, the kinetics of ZnTe MOVPE depends on the II/VI ratio: for II/VI $<5.0$, the growth rate increases with the 0.13 power of the DMZn partial pressure and with the 0.50 power of the DIPTe partial pressure; whereas for II/VI $>5.0$, the growth rate increases with the -0.13 power of the DMZn partial pressure and with the 0.75 power of the DIPTe partial pressure. These results are summarized in Table 2.

In the lower half of Figure 4, the consumption rates of DEZn and DIPTe are monitored as a function of the partial pressure of each source. On the left side (graph c), the DEZn pressure is varied, while keeping the DIPTe constant at $3.2 \times 10^{-4} \mathrm{~atm}$. Conversely, on the right side (graph d), the DIPTe pressure is varied, while keeping the DEZn constant at $4.0 \times 10^{-4} \mathrm{~atm}$. The dependence of the consumption rates of DEZn and DIPTe on their partial pressures is similar to that is observed in Figure 4a,b with DMZn and DIPTe. However, the II/VI ratio where the consumption rates of each source cross each other is equal to 0.3 instead of 5.0. Evidently, as a result of the higher reactivity of DEZn as compared to that of DMZn, the group VI compound is the limiting reagent over a much broader range of reaction conditions. Listed in Table 2 are the reaction orders for ZnTe MOVPE with DEZn and DIPTe, as determined from the slopes of the lines in Figure 4c,d. At II/VI $<0.3$, the ZnTe growth rate increases with the 0.24 power of the DEZn partial pressure and with the 0.26 power of the DIPTe partial pressure. By contrast, at II/VI $>0.3$, the ZnTe growth rate does not vary with the DEZn partial pressure but increases with the 0.60 power of the DIPTe partial pressure.

The fact that DIPTe decomposes at a faster rate than DMZn and DEZn for II/VI ratios less than 5.0 and 0.3 , respectively, is an interesting feature of ZnTe MOVPE. When the II/VI ratio is well above these values, deposits of $\mathrm{Zn}$ metal are found downstream of the reactor, as verified by X-ray diffraction. Conversely, when DIPTe is consumed in excess at II/VI ratios less than 5.0 with DMZn or 0.3 with DEZn, traces of a hazy, yellowish powder are found downstream. Under these conditions, the ZnTe film is polycrystalline ZnTe with a stoichiometric ratio of $1: 1$ as recorded by $\mathrm{X}$-ray diffraction and contains no second tellurium phase that is detectable by $\mathrm{X}$-ray microprobe analysis. Furthermore, the consumption rate of the group II precursor corresponds to the resulting film mass in this growth regime. All these data indicate that the excess DIPTe must be leaving the reactor as a volatile product. We have discovered that the DIPTe forms diisopropylditelluride (DIPDTe) and isopropyltellurol $(i$-PrTeH $)$, as described below.

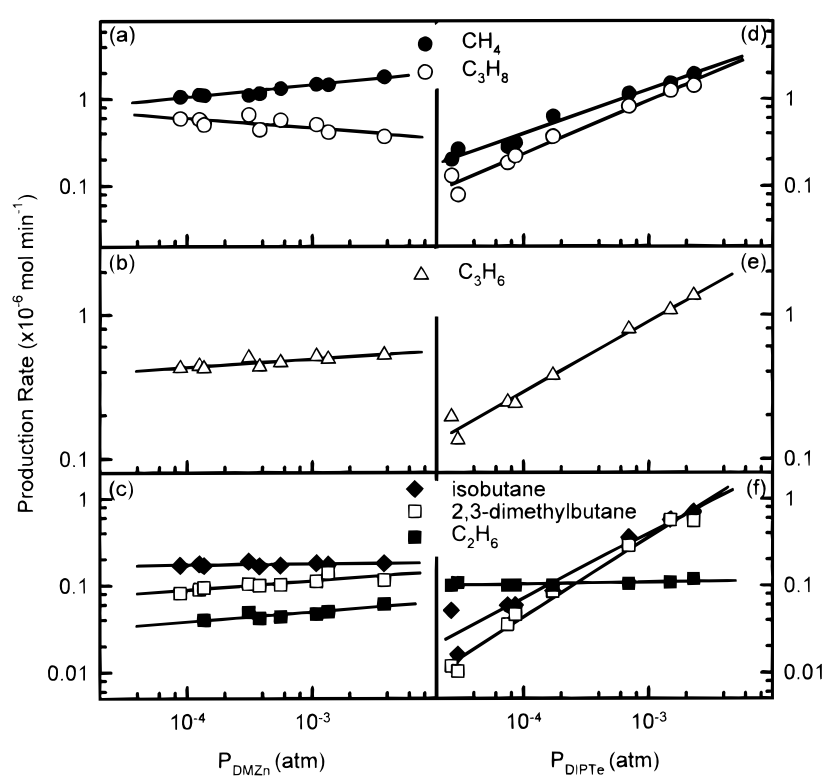

Figure 5. Dependence of the hydrocarbon production rates on the partial pressures of the sources for $\mathrm{ZnTe}$ MOVPE in $\mathrm{H}_{2}$ at $673 \mathrm{~K}$ : (ac) varying the DMZn pressure at $3.4 \times 10^{-4}$ atm of DIPTe; $(d-f)$ varying the DIPTe pressure at $4.4 \times 10^{-4}$ atm of DMZn.

Production of Hydrocarbons. The hydrocarbons produced during growth lend insight into the process by which the organometallic compounds decompose. Shown in Figure 5 is the dependence of the hydrocarbon production rates on the partial pressures of DMZn and DIPTe at $673 \mathrm{~K}$. From the top down, the figure depicts the pressure dependence of the alkyl hydrogenation products, methane and propane, the disproportionation product, propylene, and the recombination products, isobutane, 2,3-dimethylbutane, and ethane. These are the only hydrocarbons detected during growth from DMZn and DIPTe. On the left side of the figure (graphs a-c), the partial pressure of DMZn is varied, while keeping DIPTe constant at $3.4 \times$ $10^{-4} \mathrm{~atm}$. The most significant product from the decomposition of DMZn is methane. Approximately $80 \%$ of the methyl groups from DMZn are converted to methane as opposed to ethane or isobutane. The gradual increase in methane yield with the DMZn pressure tracks the DMZn consumption rate (see Figure 4). About $75 \%$ of the isopropyl groups from DIPTe are converted to propane and propylene in a nearly equivalent ratio. The propane yield decreases slightly as the partial pressure of DMZn increases, reflecting the decreasing consumption rate of DIPTe. On the other hand, the propylene yield increases a little with DMZn pressure. The rates for the recombination products (graph c) stay constant over the range of partial pressures investigated. These products contribute less than $15 \%$ of the total amount of hydrocarbons produced on a molar basis.

On the right side of Figure 5 (graphs $d-f$ ), the partial pressure of DIPTe is varied at a constant partial pressure of DMZn of $4.4 \times 10^{-4} \mathrm{~atm}$. The production of all the hydrocarbons, except ethane, increase with the partial pressure of DIPTe. This trend follows the rise in the consumption rates of both organometallic compounds (cf. Figure 4b). The dimethylzinc is again converted mostly to methane. However, at high DIPTe pressure, a significant fraction of the methyl groups are converted to isobutane. The reactions involving the recombination of isopropyl groups to form isobutane and 2,3-dimethylbutane are most sensitive to the partial pressure of the group VI compound, increasing by almost 2 orders of magnitude over the pressure range studied. At $2 \times 10^{-3}$ atm DIPTe, the recombination products account for $30 \%$ of the total hydrocarbon yield. It is interesting to note that the 2,3-dimethylbutane production rate 


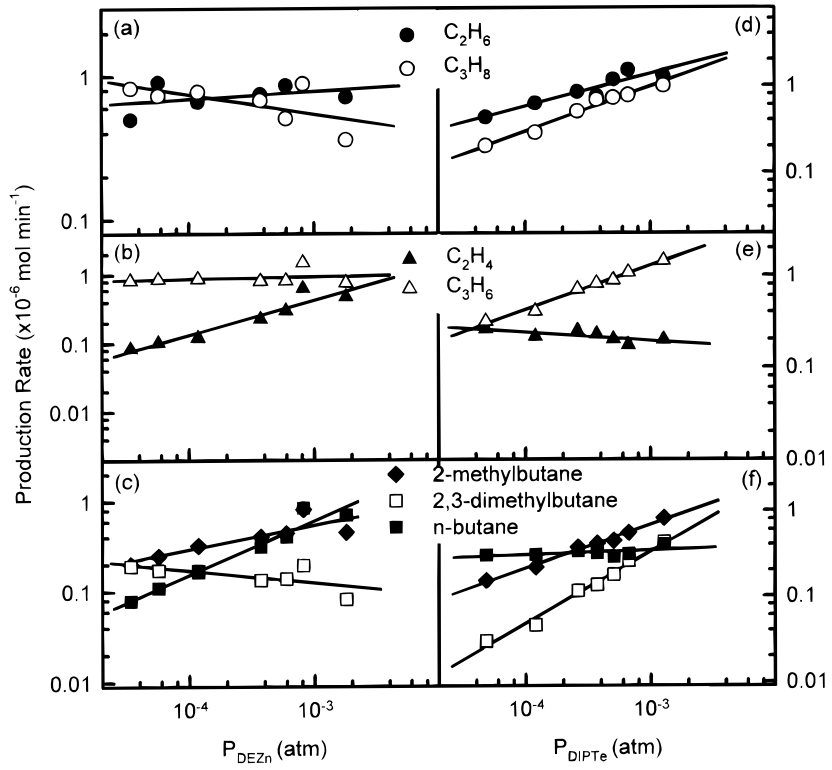

Figure 6. Dependence of the hydrocarbon production rates on the partial pressures of the sources for $\mathrm{ZnTe}$ MOVPE in $\mathrm{H}_{2}$ at $673 \mathrm{~K}$ : (ac) varying the DEZn pressure at $3.2 \times 10^{-4}$ atm of DIPTe; $(d-f)$ varying the DIPTe pressure at $4.0 \times 10^{-4} \mathrm{~atm}$ of DEZn.

overtakes the ethane production rate at a II/VI ratio of about 5.0, which is near the crossover point of the lines in Figure 4b.

Shown in Figure 6 are the effects of the source partial pressures on the formation rates of the hydrocarbons produced during MOVPE with DEZn and DIPTe. From the top down, the figure depicts the pressure dependence of the hydrogenation products, ethane and propane, the disproportionation products, ethylene and propylene, and the recombination products, 2-methylbutane, 2,3-dimethylbutane, and $n$-butane. Apart from these hydrocarbon products, trace amounts of hexane and higher hydrocarbons were detected. On the left side of the figure (graphs $\mathrm{a}-\mathrm{c}$ ), the partial pressure of DEZn is varied for a constant DIPTe pressure of $3.2 \times 10^{-4}$ atm. Unlike the high methane yields with DMZn, hydrogenation of ethyl radicals is not the main decomposition pathway for DEZn. On a molar basis, ethane comprises only $35 \%$ of the total hydrocarbons containing ethyl groups. The abundance of ethylene and $n$-butane indicates that the selectivity for disproportionation and recombination of ethyl groups is significant, especially at relatively high DEZn pressures. As the pressure of DEZn increases, the production rates of ethylene, $n$-butane, and 2-methylbutane all rise substantially. The most important hydrocarbons produced from DIPTe decomposition are propylene and propane, accounting for $75 \%$ of the isopropyl groups converted. The remainder of the isopropyl groups recombine to form 2,3-dimethylbutane.

On the right side of the Figure 6 (graphs d-f), the partial pressure of DIPTe is varied at a fixed partial pressure of DEZn of $4.0 \times 10^{-4} \mathrm{~atm}$. As the partial pressure of DIPTe increases, the production rate of all the hydrocarbons rises, except for $n$-butane and ethylene. These trends parallel the increased consumption rates of DEZn and DIPTe and, in fact, show much resemblance with the hydrocarbon yields for DMZn and DIPTe. Most of the DEZn decomposes via the hydrogenation and recombination of the ethyl groups. The hydrogenation yield increases with decreasing II/VI ratio up to a level of $\sim 50 \%$ of the total ethyl groups converted. With regard to the isopropyl radicals, a significant fraction of these are converted into recombination products, 2-methylbutane and 2,3-dimethylbutane, especially at high partial pressures of DIPTe. In Figure 5 f, the yield of 2,3-dimethylbutane intersects that of $n$-butane
TABLE 3: Effect of the $\mathrm{H}_{2}$ and He Carrier Gases on the Hydrocarbon Product Distribution for ZnTe MOVPE at 673 $\mathrm{K}$ and a II/VI Ratio of 0.25

\begin{tabular}{|c|c|c|c|c|}
\hline \multirow[b]{2}{*}{ products $^{a}$} & \multicolumn{2}{|c|}{ DMZn } & \multicolumn{2}{|c|}{ DEZn } \\
\hline & $\mathrm{H}_{2}$ & $\mathrm{He}$ & $\mathrm{H}_{2}$ & $\mathrm{He}$ \\
\hline $\begin{array}{l}\text { hydrogenation } \\
\text { methane }\end{array}$ & 0.40 & 0.36 & & \\
\hline ethane & & & 0.27 & 0.17 \\
\hline propane & 0.22 & 0.06 & 0.17 & 0.11 \\
\hline $\begin{array}{l}\text { disproportionation } \\
\text { ethylene }\end{array}$ & & & 0.03 & 0.06 \\
\hline propylene & 0.18 & 0.27 & 0.24 & 0.31 \\
\hline recombination & & & & \\
\hline ethane & 0.02 & 0.05 & & \\
\hline isobutane & 0.09 & 0.15 & & \\
\hline$n$-butane & & & 0.07 & 0.11 \\
\hline 2-methylbutane & & & 0.14 & 0.17 \\
\hline 2,3-dimethylbutane & 0.09 & 0.11 & 0.07 & 0.07 \\
\hline
\end{tabular}

at the II/VI ratio of 0.3 , which is the crossover point of the lines in Figure 4d.

A comparison of Figure 5 with Figure 6 reveals that the hydrocarbons formed from DIPTe, propane, propylene, isobutane, 2-methylbutane, and 2,3-dimethylbutane follow the same trends with the reactant partial pressure, irrespective of the group II compound used. The main difference in hydrocarbon product yields is that the methyl ligands from DMZn are converted primarily into methane, whereas the ethyl ligands from DEZn are converted into a mixture of ethane, $n$-butane, and 2-methylbutane.

Summarized in Table 3 are the effects of the zinc precursor and the carrier gas on the hydrocarbon product distribution. The deposition conditions are $673 \mathrm{~K}, 12.5 \times 10^{-4} \mathrm{~atm}$ of DIPTe, a II/VI ratio of 0.25 , and a total flow rate of $320 \mathrm{~cm}^{3} \mathrm{~min}^{-1}$. For growth with DMZn, it is observed that the fraction of methyl groups converted into methane changes little when the carrier gas is switched from hydrogen to helium. At the same time, the yield of propane decreases 4-fold, while that of propylene increases by $50 \%$. These results indicate that in He carrier gas the propyl groups supply the $\mathrm{H}$ atoms needed for hydrogenation of the methyl groups. For growth with DEZn, it is found that the fraction of alkyl groups converted into ethane and propane drops by $50 \%$ upon switching the carrier gas to helium. Conversely, the yields of ethylene, propylene, and $n$-butane increase by $50 \%$. The hydrogenation pathway is less important for the ethyl ligand compared to the methyl ligand for two reasons: (1) the former species undergoes dehydrogenation to ethylene, while the latter species cannot, and (2) recombination reactions are faster for the longer chain alkyl group. ${ }^{10}$

Production of Ditellurides and Tellurols. Below a II/VI ratio of 5.0 for MOVPE with DMZn and DIPTe and 0.3 for MOVPE with DEZn and DIPTe, the consumption rate of the group VI precursor is higher than that of the group II precursor. When this occurs, a yellowish powder deposits downstream of the reactor. This powder possesses an obnoxious odor characteristic of an organotelluride, and it turns brown soon after contact with air. Since ditellurides exhibit a yellow color and foul odor, ${ }^{24,25}$ the deposit in the reactor outlet is most likely diisopropylditelluride (DIPDTe). However, further characterization by infrared and Raman spectroscopies should confirm this.

Volatile tellurols may also be produced during MOVPE at low II/VI ratios. According to the literature, ${ }^{26-29}$ alkane tellurols and $\mathrm{H}_{2} \mathrm{Te}$ exhibit characteristic $\mathrm{Te}-\mathrm{H}$ stretching modes between 2000 and $2100 \mathrm{~cm}^{-1}$. A known method of generating tellurols is to decompose an organotelluride in hydrogen carrier gas. ${ }^{23}$ 


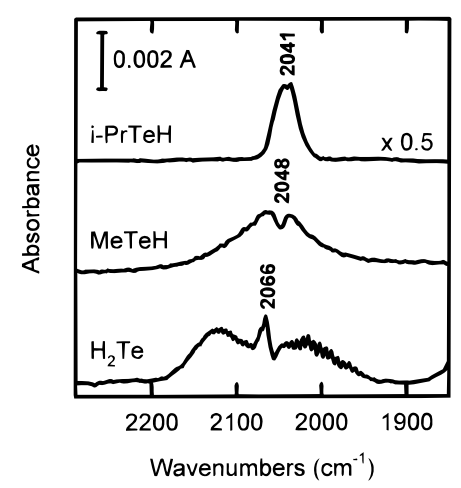

Figure 7. Infrared spectra of the $\mathrm{Te}-\mathrm{H}$ stretching vibration for isopropyltellurol, methyltellurol, and hydrogen telluride.

Accordingly, infrared spectra of the effluent gas from the reactor during the pyrolysis of DIPTe and DMTe in $\mathrm{H}_{2}$ have been collected at different temperatures. Tellurium hydride stretching vibrations for isopropyltellurol $(i$-PrTeH), methyltellurol (Me$\mathrm{TeH})$, and hydrogen telluride $\left(\mathrm{H}_{2} \mathrm{Te}\right)$ were observed in these experiments. These infrared spectra are reproduced in Figure 7. Hydrogen telluride is produced by pyrolysis of DIPTe at $873 \mathrm{~K}$. The spectrum consists of a sharp Q-branch at 2066 $\mathrm{cm}^{-1}$ with broad P- and R-branches on either side. The $\mathrm{Te}-\mathrm{H}$ mode is also accompanied by low-frequency bands at 872 (broad) and $839 \mathrm{~cm}^{-1}$ (sharp). These features coincide well with those found previously by Hamada. ${ }^{28}$

During the pyrolysis of DIPTe at $723 \mathrm{~K}$, the top spectrum in Figure 7 is observed along with a low-intensity spectrum for $\mathrm{H}_{2}$ Te. After subtraction of the $\mathrm{H}_{2} \mathrm{Te}$ absorbances, the relatively narrow doublet remains at $2041 \mathrm{~cm}^{-1}$. This feature can be assigned to that of isopropyltellurol, since it is accompanied by the isopropyl skeletal modes at 1208 and $1157 \mathrm{~cm}^{-1}$ and the $\mathrm{Te}-\mathrm{C}$ stretching vibration at $642 \mathrm{~cm}^{-1}$. Pyrolysis of dimethyltelluride alone at $873 \mathrm{~K}$ yields a spectrum of $\mathrm{H}_{2} \mathrm{Te}$ and methane. However, at $723 \mathrm{~K}$, a new doublet appears centered at $2048 \mathrm{~cm}^{-1}$, which closely resembles the spectrum of MeTeH reported in the literature. ${ }^{26}$ The middle spectrum in Figure 7 shows this feature after the $\mathrm{H}_{2} \mathrm{Te}$ spectrum has been subtracted out. The other infrared bands of methyltellurol could not be resolved due to their overlap with the intense bands of methane. Other than the $v_{\mathrm{Te}-\mathrm{H}}$ vibrational band of hydrogen telluride, the spectra of methyltellurol and isopropyltellurol are more narrow and do not show any rotational fine structure or the central Q-branch. This is expected for heavier molecules with asymmetrical ligands. ${ }^{19,22}$ Our assignment of the vibrational bands is also consistent with the frequency shift observed from $2066 \mathrm{~cm}^{-1}$ for $\mathrm{H}_{2} \mathrm{Te}$, to $2048 \mathrm{~cm}^{-1}$ for $\mathrm{MeTeH}$, to $2041 \mathrm{~cm}^{-1}$ for $i$-PrTeH. It is well-known that when the size of the ligands on the central (M) atom increases, the $\mathrm{M}-\mathrm{H}$ stretching vibration shifts to lower frequency. For example, in the series $\mathrm{H}_{2} \mathrm{O}, \mathrm{CH}_{3}-$ $\mathrm{OH}$, and $\mathrm{C}_{3} \mathrm{H}_{7} \mathrm{OH}$, the isolated $\mathrm{OH}$ stretching frequencies recorded in the gas phase are 3740,3681 , and $3636 \mathrm{~cm}^{-1}$, respectively. ${ }^{28,30}$

It should be noted that no yellow deposit is detected downstream of the reactor following the pyrolysis of DIPTe and DMTe. If any ditellurides are formed, their concentration must be quite small. Consequently, the amount of tellurol produced during pyrolysis must be equal to the amount of DIPTe consumed minus the amount of solid tellurium deposited. Based on measuring these amounts, the spectra in Figure 7 for $\mathrm{H}_{2} \mathrm{Te}$ and $i$-PrTeH correspond to about 200 and $100 \mathrm{ppm}$, respectively, of these molecules in the effluent gas. (The MeTeH spectrum was not calibrated.)

During the deposition of ZnTe from DMZn, or DEZn, and DIPTe at low II/VI ratios, the absorption band at $2041 \mathrm{~cm}^{-1}$

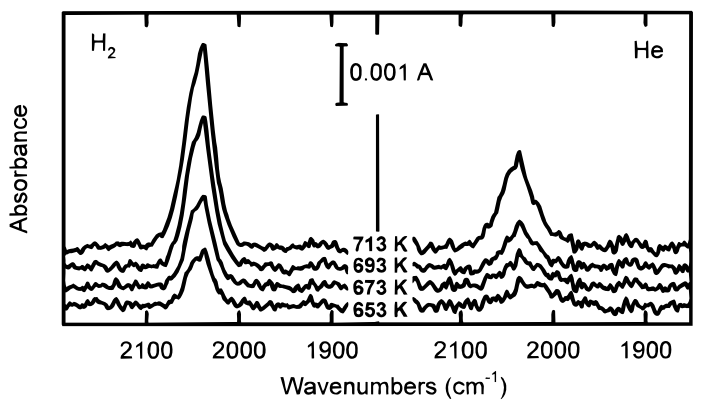

Figure 8. Infrared spectra of the $\mathrm{Te}-\mathrm{H}$ stretching mode for isopropyltellurol in the effluent gas for ZnTe MOVPE at different temperatures and in $\mathrm{H}_{2}$ or $\mathrm{He}$ carrier gas. The II/VI ratio is 0.25 , and the DMZn partial pressure is $5 \times 10^{-4} \mathrm{~atm}$.

for the isopropyltellurol is observed. No other bands characteristic of $\mathrm{MeTeH}$ or $\mathrm{H}_{2} \mathrm{Te}$ are detected. Shown in Figure 8 are the spectra of $i$-PrTeH during ZnTe MOVPE from DMZn and DIPTe in hydrogen and helium carrier gases. Evidently, more $i$ - $\mathrm{PrTeH}$ is produced in $\mathrm{H}_{2}$ than in $\mathrm{He}$, and the concentration of this product increases linearly with temperature. In addition, it is observed that the amount of isopropyltellurol produced is a linear function of the amount of DIPTe consumed in excess of DMZn. The band in the spectrum during growth in hydrogen at $719 \mathrm{~K}$ corresponds to about $40 \mathrm{ppm}$ of $i$-PrTeH or $10 \%$ of the DIPTe consumed in excess of the DMZn.

A mass balance is presented in Table 4 for $\mathrm{ZnTe}$ MOVPE with DMZn and DIPTe at $673 \mathrm{~K}$ and a II/VI ratio of 0.25 . The DMZn and DIPTe consumption rates were determined by monitoring the change in intensities of the infrared bands. The $\mathrm{ZnTe}$ growth rate was estimated by weighing the film after the run. This growth rate agreed to within $10 \%$ of that estimated from the DMZn consumption rate. The production rate of $i$-PrTeH was calculated from the intensity of the $\mathrm{Te}-\mathrm{H}$ vibrational band. Only the production rate of DIPDTe was not directly measured. However, the amount of hydrocarbons produced from DIPTe as measured by GC are much lower than the expected amount based on the consumption rate of DIPTe from IR. This difference is attributed to the production of $i$-PrTeH and DIPDTe, which are not detected by GC. An estimate of the DIPDTe production rate may be obtained from a mass balance on the propyl groups:

$$
\begin{array}{r}
r_{\text {DIPDTe }}(i-\mathrm{Pr})=1 / 2\left(2 r_{\mathrm{DIPTe}}-r_{\mathrm{C}_{3} \mathrm{H}_{8}}-r_{\mathrm{C}_{3} \mathrm{H}_{6}}-r_{\mathrm{C}_{4} \mathrm{H}_{10}}-\right. \\
\left.2 r_{\mathrm{CH}_{14}}-r_{i-\mathrm{PrTeH}}\right)
\end{array}
$$

where $r$ is the rate, and the subscripts refer to the individual components $\left(\mathrm{C}_{3} \mathrm{H}_{8}=\right.$ propane, $\mathrm{C}_{3} \mathrm{H}_{6}=$ propylene, $\mathrm{C}_{4} \mathrm{H}_{10}=$ isobutane, and $\mathrm{C}_{6} \mathrm{H}_{14}=2,3$-dimethylbutane). Alternatively, the DIPDTe production rate may be calculated from a mass balance on tellurium:

$$
r_{\text {DIPDTe }}(\mathrm{Te})={ }_{1}^{1}\left(r_{\mathrm{DIPTe}}-r_{\mathrm{ZnTe}}-r_{\mathrm{i}-\mathrm{PrTeH}}\right)
$$

The production rate of diisopropyl ditelluride determined from eqs 2 and 3 are shown in Table 4 for ZnTe MOVPE in $\mathrm{H}_{2}$. The agreement is excellent.

\section{Discussion}

Growth Kinetics of ZnTe MOVPE. By monitoring the inlet and outlet gases of the MOVPE reactor with infrared spectroscopy and gas chromatography, we have made a number of important discoveries about the reaction chemistry. We find that the decomposition rates of the group II and VI sources exhibit different kinetics. Depending on the II/VI ratio, one of 
TABLE 4: Reaction Rates Recorded during ZnTe MOVPE with DMZn and DIPTe at $673 \mathrm{~K}$, a II/VI Ratio of 0.25 , and a DMZn Partial Pressure of $3.5 \times 10^{-4} \mathrm{~atm}^{a}$

\begin{tabular}{ccc}
\hline & \multicolumn{2}{c}{ rate $\left(\times 10^{-6} \mathrm{~mol} \mathrm{~min}^{-1}\right)$} \\
\cline { 2 - 3 } compound & $\mathrm{H}_{2}$ & $\mathrm{He}$ \\
\hline reactant & & \\
DIPTe & 2.35 & 1.82 \\
DMZn & 1.16 & 0.79 \\
product & & \\
ZnTe & 1.04 & $\mathrm{~nm}^{c}$ \\
$i$-PrTeH & 0.12 & 0.05 \\
DIPDTe & $0.49^{a}$ & $0.39^{a}$ \\
& $0.54^{b}$ & $0.49^{b}$
\end{tabular}

${ }^{a}$ From eq 2. ${ }^{b}$ From eq 3, assuming $r_{\mathrm{ZnTe}}=r_{\mathrm{DMZn} \cdot} \cdot{ }^{c}$ Not measured.

the sources reacts at a slower rate than the other one. The slowest rate determines the rate of $\mathrm{ZnTe}$ film growth. The faster reacting source produces waste byproducts: $\mathrm{Zn}$ in the case of DMZn and DEZn and $i$-PrTeH and DIPDTe in the case of DIPTe. Since the excess $\mathrm{Zn}$ and Te reaction products are volatile, a stoichiometric $\mathrm{ZnTe}$ film is deposited under all reaction conditions. The conditions under which the consumption rate of the group II equals that of the group VI source depends on the reactivity of the organometallic compounds. For ZnTe MOVPE at $673 \mathrm{~K}$ using DMZn and DIPTe, the crossover point of the consumption rates occurs at a relatively high II/VI ratio of 5.0, whereas for DEZn and DIPTe the crossover point occurs at a II/VI ratio of 0.3 . These results may be compared to CdTe MOVPE with DMCd and DIPTe, where the crossover point of the consumption rates occurs at a II/VI ratio below 0.5 (ref 10).

The kinetics presented in Table 2 are consistent with the heterogeneous decomposition of the two sources.,7,10 The apparent activation energies and the orders in the reactant pressures can be explained by a Langmuir-Hinshelwood mechanism in which a surface reaction is the rate-limiting step. The near zero-order dependence on the DMZn and DEZn pressures suggests that the coverage of these species are relatively high under MOVPE conditions. Conversely, the reaction orders for DIPTe range from 0.26 to 0.75 , indicative of an intermediate coverage of this species. Further examination of Table 2 reveals that the reaction order is significantly lower when a particular source is in excess than when it is the limiting reagent. For example, the reaction order in DEZn is 0.24 when DIPTe decomposes faster than DEZn but is -0.02 when the reverse is true. This implies that the adsorption sites are saturated with ethyl groups at the crossover point where DEZn becomes the excess reagent.

Reaction Pathways for Alkyl Ligands. The hydrocarbon product distribution can be explained by radical-like surface reactions of the alkyl ligands. However, it is also possible that some fraction of the alkyl groups desorb as radicals and react to form stable alkanes in the gas. ${ }^{10}$ A good indicator of radical chemistry is the ratio of the rate constants for olefin formation to alkane recombination $\left(k_{\mathrm{d}} / k_{\mathrm{r}}\right){ }^{10,31,32}$ If the ratio of the rate constants is less than or equal to unity, then this is consistent with radical chemistry, in which the olefin is produced by disproportionation. If, on the other hand, the $k_{\mathrm{d}} / k_{\mathrm{r}}$ ratio is much greater than one, then $\beta$-hydride elimination is likely to dominate. This latter reaction is common on metal surfaces. ${ }^{32}$ The $k_{\mathrm{d}} / k_{\mathrm{r}}$ ratio is calculated from the hydrocarbon production distribution. For MOVPE with DMZn and DIPTe, only the isopropyl groups can disproportionate. The ratio in this case is given by

$$
k_{\mathrm{d}} / k_{\mathrm{r}}(i-\mathrm{Pr})=y_{\mathrm{C}_{3} \mathrm{H}_{6}} /\left(2 y_{\mathrm{C}_{6} \mathrm{H}_{14}}+y_{\mathrm{C}_{4} \mathrm{H}_{10}}\right)
$$

where $y$ is the product yield. From Table $3, k_{\mathrm{d}} / k_{\mathrm{r}}(\mathrm{i}-\mathrm{Pr})$ equals 0.67 in $\mathrm{H}_{2}$ and 0.73 in He. For MOVPE with DEZn and DIPTe, the ethyl and isopropyl groups can disproportionate so that two ratios may be calculated:

$$
\begin{gathered}
k_{\mathrm{d}} / k_{\mathrm{r}}(\mathrm{Et})=y_{\mathrm{C}_{2} \mathrm{H}_{4}} /\left(2 y_{\mathrm{C}_{4} \mathrm{H}_{10}}+y_{\mathrm{C}_{5} \mathrm{H}_{12}}\right) \\
k_{\mathrm{d}} / k_{\mathrm{r}}(i \text { - } \mathrm{Pr})=y_{\mathrm{C}_{3} \mathrm{H}_{6}} /\left(2 y_{\mathrm{C}_{6} \mathrm{H}_{14}}+y_{\mathrm{C}_{5} \mathrm{H}_{12}}\right)
\end{gathered}
$$

From Table $3, k_{\mathrm{d}} / k_{\mathrm{r}}(\mathrm{Et})$ equals 0.11 in $\mathrm{H}_{2}$ and 0.15 in $\mathrm{He}$, whereas $k_{\mathrm{d}} / k_{\mathrm{r}}(i-\mathrm{Pr})$ is 0.85 in $\mathrm{H}_{2}$ and 1.0 in He. These calculation clearly indicate that the alkyl reactions are consistent with radical chemistry.

During ZnTe MOVPE with DMZn and DIPTe, $75 \%$ of the group II compound decomposes through hydrogenation to form methane. As is evident in Table 3, the methane production decreases only slightly when the carrier gas is switched from hydrogen to helium. In the helium ambient, hydrogenation reactions must occur on the film surface, where the necessary hydrogen atoms are provided by disproportionation of the isopropyl groups. In other words, the majority of the methyl groups from DMZn leave the surface as methane instead of methyl radicals. The ethyl groups from DEZn, on the other hand, are converted into a variety of products consistent with radical hydrogenation, disproportionation, and recombination. The different reaction pathways exhibited by the methyl and ethyl ligands are probably due to the higher $\mathrm{M}-\mathrm{C}$ bond strength of DMZn compared to that of DEZn. The average bond dissociation energies of these molecules are 176 and $146 \mathrm{~kJ}$ mol $^{-1}$, respectively. ${ }^{5}$ It should be noted that DMCd, which has a bond dissociation energy similar to DEZn, also exhibits a hydrocarbon product distribution consistent with radical surface chemistry. ${ }^{10}$

The surface hydrogenation of methyl groups into methane is contrary to current understanding of compound semiconductor surface chemistry. Vohs and co-workers ${ }^{33}$ have investigated the decomposition of DMZn on $\mathrm{GaAs}(001)$ and have only detected methyl radicals and zinc atoms with the mass spectrometer during temperature-programmed desorption. Furthermore, it is generally agreed that trimethylgallium decomposes by desorption of methyl radicals on $\mathrm{GaAs}(001)$ surfaces. ${ }^{34,35}$ The discrepancy between our observations and the surface science studies may be due to the drastically different conditions under which the experiments are performed. In the latter case, the organozinc compound reacts by itself without any other species present, whereas under MOVPE conditions, the group II or VI compounds are decomposing side by side on the film surface and in the presence of an enormous incoming flux of these reagents.

Production of Ditellurides and Tellurols. This is the first report on the production of diisopropylditelluride and isopropyltellurol as waste byproducts during $\mathrm{ZnTe}$ MOVPE. Under the conditions studied herein, more than $80 \%$ of the excess DIPTe is converted into diisopropylditelluride (see Table 4). The DIPDTe is most likely formed by the recombination of two adsorbed $i$-PrTe groups, whereas the isopropyltellurol is probably generated by the recombination of adsorbed $i$-PrTe and $\mathrm{H}$. The tellurol is also produced in significant quantity during DIPTe pyrolysis, which suggests that it could arise from gas-phase reactions as well.

\section{Conclusions}

During ZnTe MOVPE from DMZn, or DEZn, and DIPTe, two growth regimes exist which are determined by the II/VI ratio. In one regime, the DIPTe is consumed in excess, whereas 
in the other regime, the group II compound is consumed in excess. The decomposition rate of the limiting reagent determines the ZnTe growth rate. Volatile waste byproducts are produced from whichever source decomposes at a faster rate: Zn from DMZn and DEZn and DIPDTe and $i$-PrTeH from DIPTe. The group II and VI sources exhibit different rate expressions for decomposition which do not depend on the II/ VI ratio. However, the growth kinetics do depend on the II/VI ratio, because they follow the kinetics of the rate-limiting source.

The rate laws for the decomposition of the organometallic sources are consistent with a heterogeneous mechanism, in which the rate-limiting step involves a surface reaction of the adsorbed precursor. The hydrocarbon products also appear to be formed by surface reactions of the alkyl ligands. In the case of the methyl groups, the dominant pathway is hydrogenation to form methane. In contrast, the ethyl and propyl groups undergo "radical-like" reactions comprising of hydrogenation, disproportionation, and recombination.

Acknowledgment. We express our gratitude to the U.S. Environmental Protection Agency, the National Science Foundation, the UC MICRO program, and Morton Metalorganics for their generous support of this work. In addition, we are grateful for the National Science Foundation Graduate Fellowship that was awarded to K.J.W.

\section{References and Notes}

(1) Kisker, D. W. J. Cryst. Growth 1989, 98, 127.

(2) Kuhn, W. S.; Qu'Hen, B.; Gorochev, O. Prog. Cryst. Growth Charact. 1995, 31, 144.

(3) Kuhn, W. S.; Qu'Hen, B.; Gorochev, O.; Tribulet, R.;Gebhardt, W. Prog. Cryst. Growth Charact. 1995, 31, 45-117.

(4) Taniguchi, S.; Hino, T.; Itoh, S.; Nakano, K.; Nakayama, N.; Ishibashi, A.; Ikeda, M. Electron. Lett. 1996, 32, 552.

(5) Stringfellow, G. B. Organometallic Vapor-Phase Epitaxy, Theory and Practice; Academic Press: New York, 1989.

(6) Bloomfield, C. Compd. Semicond. 1995, 1, 32.

(7) Irvine, S. J. C.; Bajaj, J. J. Cryst. Growth 1994, 145, 74. 55,92 .
(9) Nishio, M.; Ogawa, H.; Yoshida A. Vacuum 1990, 41, 715.

(10) McDaniel, A. H.; Wilkerson, K. J.; Hicks, R. F. J. Phys. Chem. 1995, 99, 3574 .

(11) Ahlgren, W. L.; Johnson, S. M.; Smith, E. J.; Ruth, R. P.; Johnston, M. H.; Kalisher, C.; Cockrum, A.; James, T. W.; Arney, D. L.; Ziegler, C. K.; Lick, W. J. Vac. Sci. Technol. 1989, A7, 331.

(12) Hails, J. E.; Irvine, S. J. C. J. Cryst. Growth 1991, 107, 319.

(13) Bell, W.; McQueen, A. E. D.; Walton, J. C.; Foster, D. F.; ColeHamilton, D. J.; Hails, J. E. J. Cryst. Growth 1992, 117, 58.

(14) McQueen, A. E. D.; Culshaw, P. N.; Walton, J. C.; ShenaiKhatkhate, D. V.; Cole-Hamilton, D. J.; Mullin, J. B. J. Cryst. Growth 1991, 107, 325 .

(15) Czerniak M. R.; Easton, B. C. J. Cryst. Growth 1984, 68, 128.

(16) Bhat, I. B.; Taskar, N. R.; Ghandhi, S. K. J. Electrochem. Soc. 1987, $134,195$.

(17) Butler, I. S.; Newbury, M. L. Spectrochim. Acta 1977, 33A, 669.

(18) Kaesz, H. D.; Stone, F. G. A. Spectrochim. Acta 1959, 360.

(19) Bellamy, L. J. The Infra-red Spectra of Complex Molecules; Wiley: New York, 1956.

(20) Joint Committee on Atomic and Molecular Physical Data, Evaluated Infrared Reference Spectrum; Coblentz Society: Philadelphia, 1973.

(21) Pescok, R. L.; Shields, L. D. Modern Methods of Chemical Analysis; Wiley: New York, 1968.

(22) Herzberg, G. Molecular Spectra and Molecular Structure: Infrared and Raman Spectra of Polyatomic Molecules; Van Nostrand Reinhold: New York, 1945.

(23) Kirss, R. U.; Brown, D. W.; Higa, K. T.; Gedridge, R. W., Jr Organometallics 1991, 10, 3589.

(24) Irgolic, K. J. The Organic Chemistry of Tellurium; Gordon: New York, 1974.

(25) Klayman, D. L.; Gunter, W. H. H. Organic Selenium Compounds: Their Chemistry and Biology; Wiley-Interscience: New York, 1973.

(26) Sink, C. W.; Harvey, A. B. J. Chem. Phys. 1972, 57, 4434.

(27) Hamada, K.; Morishita, H. Synth. React. Inorg. Met.-Org. Chem. 1977, 7, 355 .

(28) Hamada, K. Spectrosc. Lett. 1987, 20, 537.

(29) Dabbousi, B. O.; Bonasia, P. J.; Arnold, J. J. Am. Chem. Soc. 1991, $113,186$.

(30) Lutz, E. T. G. Private communications.

(31) Lin, J.; Bent, B. E. J. Am. Chem. Soc. 1993, 115, 6943.

(32) Paul, A.; Yang, M. X.; Bent, B. E. Surf. Sci. 1993, 297, 327.

(33) Venkateswaran, N.; Roe, C. L.; Lam, H.-T.; Vohs, J. M. Surf. Sci. 1996, 365, 125.

(34) Creighton, J. R. Surf. Sci. 1990, 234, 287.

(35) Creighton, J. R.; Banse, B. A. Mater. Res. Soc. Symp. Proc. 1991, 222,15 . 